\title{
Unveiling the role of the lone electron pair in sesquioxides at high pressure: Compressibility of $\beta-\mathrm{Sb}_{2} \mathrm{O}_{3}$
}

Received 00th January 20xx, Accepted 00th January 20xx DOI: $10.1039 / x 0 x \times 00000 x$
Juan Angel Sans, ${ }^{a,}{ }^{*}$ Francisco Javier Manjón, ${ }^{a}$ André Luis de Jesus Pereira, ${ }^{a, b}$ Javier Ruiz-Fuertes, ${ }^{c}$ Catalin Popescu, ${ }^{d}$ Alfonso Muñoz, ${ }^{e}$ Plácida Rodríguez-Hernández, ${ }^{\text {e }}$ Julio Pellicer-Porres, ${ }^{f}$ Vanesa

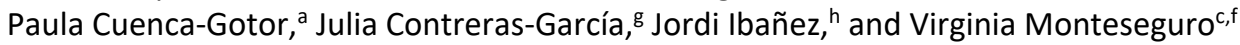

\begin{abstract}
The structural, vibrational and electronic properties of the compressed $\beta-\mathrm{Sb}_{2} \mathrm{O}_{3}$ polymorph, a.k.a. mineral valentinite, have been investigated in a joint experimental and theoretical study up to $23 \mathrm{GPa}$. The compressibility of the lattice parameters, unit-cell volume and polyhedral unit volumes as well as the behaviour of its Raman- and IR-active modes under compression have been interpreted on the basis of ab initio theoretical simulations. Valentinite shows an unusual compressibility up to 15 GPa showing four different pressure ranges, whose critical pressures are 2, 4, and $10 \mathrm{GPa}$. The pressure dependence of the main structural units, the lack of soft phonons, and the electronic density charge topology consigns the changes at those critical pressures to isostructural phase transitions of degree higher than 2. In particular, the transitions at 2 and 4 GPa can be ascribed to changes in the interaction between the stereochemically-active lone electron pairs of $\mathrm{Sb}$ atoms under compression. The changes observed above $10 \mathrm{GPa}$, characterized by a general softening of several Raman- and IR-active modes, point to a structural instability prior of the 1st-order transition occurring above $15 \mathrm{GPa}$. Above this pressure, a tentative new high-pressure phase (s.g. Pcc2) has been assigned by single-crystal and powder X-ray diffraction measurements.
\end{abstract}

\section{Introduction}

The study of the different polymorphs of group-XV sesquioxides (valence $3+$ ) has triggered the interest of the scientific community in the last years [1-3]. Recent studies have predicted the possible structures of their different polymorphs by the analysis on their dynamical stability. Thus, their structures can be understood on the basis of deformations of the original defective-fluorite structure $[4,5]$ by applying a vacant array along different crystallographic directions.

The different polymorphs of these sesquioxides are likely related to the distortion of the charge density produced by the cationic lone electron pair (LEP), belonging to the outer $n s^{2}$ electrons, when there is a strong $s-p$ hybridization between the cation and the anion [6-8]. However, despite the efforts that the

-Instituto de Diseño para la Fabricación y Producción Automatizada, MALTA Consolider Team, Universitat Politècnica de València, 46022 València, Spain b. Grupo de Pesquisa em Materiais Fotonicos e Energia Renovável - MaFER,

Faculdade de Ciências Exatas e Tecnologia, Universidade Federal da Grande Dourados, Dourados, Brazil

c. DCITIMAC, MALTA Consolider Team, Universidad de Cantabria, Santander 39005, Spain

ALBA-CELLS, 08290 Cerdanyola del Vallés, Barcelona, Spain

e. Departamento de Física, Instituto de Materiales y Nanotecnología, MALTA Consolider Team, Universidad de La Laguna, 38207 San Cristóbal de La Laguna, Spain

f. Departamento de Física Aplicada-ICMUV, MALTA-Consolider Team, Universitat de València, València, Spain

g. CNRS, UMR 7616, Laboratoire de Chimie Théorique, F-75005, Paris, France

h. Institute of Earth Sciences Jaume Almera, CSIC, 08028 Barcelona, Spain

Electronic Supplementary Information (ESI) available: [details of any supplementary information available should be included here]. See DOI: 10.1039/x0xx00000x scientific community has put to understand the structural stability and formation of the different polymorphs and new phases of these sesquioxides [9], the exact role played by the cationic LEP in the different polymorphs remains an open question. It is known that when the distortions are strong enough, the LEP effect can force the structures to form closedmolecular units separated one to each other. This is the case of the most symmetric cubic structures of minerals arsenolite (c$\left.\mathrm{As}_{2} \mathrm{O}_{3}\right)$ and senarmontite $\left(\alpha-\mathrm{Sb}_{2} \mathrm{O}_{3}\right)$, where the large influence of the LEP stabilizes a structure with a large number of voids. The behaviour of arsenolite and senarmontite has been recently studied under compression [10-14]. While arsenolite shows no pressure-induced transition, a high-pressure (HP) phase of senarmontite has been recently elucidated and labelled alphaII [space group (s.g.) $114, P \overline{4} 2_{1} c, \mathrm{Z}=4$ ], being structurally quite similar to tetragonal $\beta-\mathrm{Bi}_{2} \mathrm{O}_{3}[14]$.

The less symmetric structures of group-XV sesquioxides and the behaviour of the cationic LEP in these compounds have also been studied at HP. In particular, the polymorphs belonging to the monoclinic symmetry $\alpha-\mathrm{Bi}_{2} \mathrm{O}_{3}\left(\beta-\mathrm{As}_{2} \mathrm{O}_{3}\right)$, a.k.a. mineral bismite (claudetite I) have revealed a large structural stability at HP up to $20(40) \mathrm{GPa}[\mathbf{1 5}, \mathbf{1 6}]$. A different behaviour has been observed in another monoclinic phase of $\mathrm{As}_{2} \mathrm{O}_{3}$, a.k.a. claudetite II, that shows a pressure-induced isostructural phase transition (IPT) above 2 GPa [17].

Finally, sesquioxides with intermediate symmetric structures (tetragonal and orthorhombic), like $\beta-\mathrm{Bi}_{2} \mathrm{O}_{3}, \varepsilon-\mathrm{Bi}_{2} \mathrm{O}_{3},[18]$ and $\beta$ $\mathrm{Sb}_{2} \mathrm{O}_{3}$ are not so well studied at HP despite they can be the key structures to understand the cationic LEP activity and its 
contribution to HP polymorphs. In these last polymorphs, the LEP effect triggers the formation of empty cavities arranged along a certain crystallographic direction. Among these compounds, only the HP behaviour of $\beta-\mathrm{Bi}_{2} \mathrm{O}_{3}$ has been thoroughly studied; giving rise to a $2^{\text {nd }}$ order ferroelastic IPT above 2 GPa caused by an electronic charge redistribution around the $\mathrm{BiO}_{6}$ polyhedra $[\mathbf{1 8 , 1 9 ]}$. Since the cationic LEP stereoactivity is more intense in compounds with smaller unitcell volumes [20], sesquioxides formed with cations with smaller ionic radius than $\mathrm{Bi}$ ( $\mathrm{As}$ or $\mathrm{Sb}$ ) should exhibit a larger influence of the LEP. Thus, the HP study of orthorhombic $\beta$ $\mathrm{Sb}_{2} \mathrm{O}_{3}$ with similar empty linear channels as $\beta-\mathrm{Bi}_{2} \mathrm{O}_{3}$, can be very interesting to uncover the role played by the LEP in the formation of the different group-XV sesquioxide polymorphs and understand their pressure-induced transformations.

In particular, the $\mathrm{Sb}_{2} \mathrm{O}_{3}$ compound is used as a retardant in a number of materials [21,22], as oxidation catalyst in several production processes $[\mathbf{2 3}, \mathbf{2 4}]$, as anode material for $\mathrm{Li}$ - and $\mathrm{Na}$ ion batteries $[\mathbf{2 5}, \mathbf{2 6}]$, to increase stability and decrease wear in fluid lubricants [27], to enhance the properties of glasses [28,29], and also as dopant to modify the conductivity of semiconductors, like $\mathrm{SnO}_{2}$ and $\mathrm{ZnO}$ [30-33]. Furthermore, the synthesis, properties, and applications of nanoparticles of antimony oxides have been recently reviewed since nanoparticles possess excellent properties as compared to bulk material, like a higher refractive index, absorbability, abrasive resistance, and proton conductivity [34].

In the literature, previous HP Raman scattering (RS) $[35,36]$ and an X-ray diffraction (XRD) studies [37], have shown that $\beta-\mathrm{Sb}_{2} \mathrm{O}_{3}$ undergoes a structural phase transition above $15 \mathrm{GPa}$. While those works have studied the overall compression of valentinite up to $40 \mathrm{GPa}$, showing the presence of two polymorphs up to that pressure, they have not carefully studied the behaviour of the low-pressure (LP) phase of $\beta-\mathrm{Sb}_{2} \mathrm{O}_{3}$ prior to the structural phase transition to the HP phase, that was proposed to be of monoclinic symmetry [37]. This has motivated us to perform a detailed experimental and theoretical study of the structural, vibrational and electronic properties of $\beta-\mathrm{Sb}_{2} \mathrm{O}_{3}$ under compression with a thorough analysis of the theoretical electronic charge topology of the LP phase. We will show that compressed valentinite reveals several internal transitions, prior to its structural phase transition above $15 \mathrm{GPa}$. Most of these internal transitions will be shown to be related to changes in the cationic LEP under compression and could be considered as IPTs of order higher than 2. The incompleteness of the Landau and Liftshitz's description of solid phase transitions [38] was already stated by Liftshitz in 1960 with the inclusion of the electronic topological transitions (ETTs) as transitions of order $2^{1 / 2}$ (higher than 2) [39] according to the Ehrenfest's notation [40]. The $2^{\text {nd }}$ order phase transitions requires a soft mode, according to Landau's theory; however, the changes observed in this work are associated to changes in the interaction of the cationic lone electron pairs, i. e. with an electronic origin such as the one observed in ETTs, which are ascribed to transitions of order higher than 2. Recent studies [41] have proved the existence of IPTs of order higher than 1 (continuous), some of them associated with changes in the electronic topology.
Therefore, this work shows the importance of the cationic LEP activity in relation to the rest of electrons in the molecular unit in order to understand the properties of this quasi-molecular material. Moreover, thanks to the combination of single-crystal and powder XRD measurements, we will propose an orthorhombic HP phase [s.g. Pcc2] above $15 \mathrm{GPa}$, that is different to the monoclinic HP phase previously proposed [37]. Consequently, this study allows completing the understanding of the polymorphism of group XV sesquioxides and becomes interesting for the future analysis of other LEP-based molecular and quasi-molecular solids under extreme conditions.

\section{Results}

\section{Structural properties of $\beta-\mathrm{Sb}_{2} \mathrm{O}_{3}$ under compression}

The structure of $\beta-\mathrm{Sb}_{2} \mathrm{O}_{3}$ [s.g. 56, Pccn, $\mathrm{Z}=4$ ] at room pressure has been traditionally interpreted as formed by $\mathrm{SbO}_{3} E$ units (see the $\mathrm{SbO}_{3}$ polyhedral unit at the bottom part of Fig. 1), where the $E$ refers to the cationic LEP. The link of different $\mathrm{SbO}_{3} E$ units along the c-axis (two-fold rotation axis) form rods composed by infinite $\left(\mathrm{Sb}_{2} \mathrm{O}_{3}\right)_{\infty}$ chain molecules in such a way that neighbour LEPs form empty linear channels parallel to the rods. In this context, $\beta-\mathrm{Sb}_{2} \mathrm{O}_{3}$ can be considered as a quasi-molecular solid (with infinite molecules along the c-axis) or an acicular solid since it grows in form of $\left(\mathrm{Sb}_{4} \mathrm{O}_{6}\right)_{\infty}$ needles extended along the c-axis (see Fig. 1).

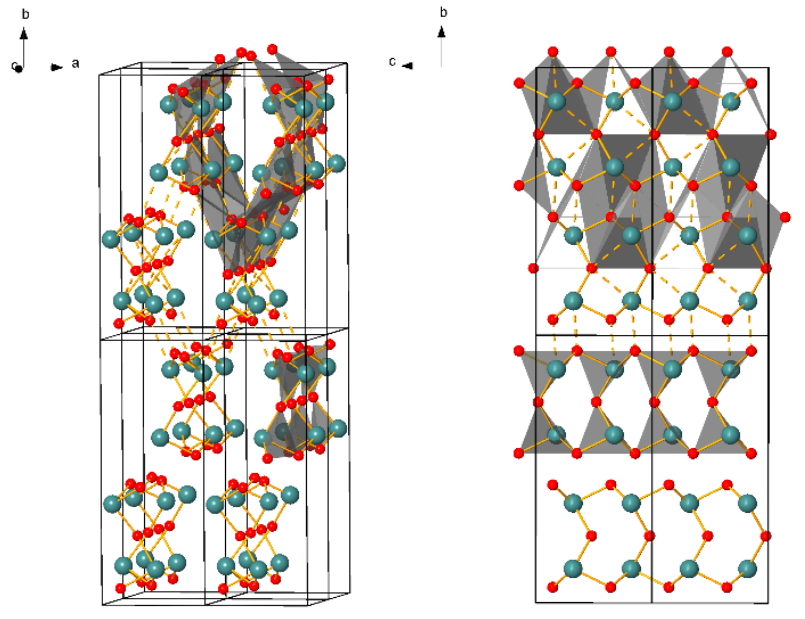

Figure 1. (left) 3D structure layout of the low-pressure (LP) phase of $\beta-\mathrm{Sb}_{2} \mathrm{O}_{3}$ at ambient pressure (labelling Sb-cyan, O1-red and O2-orange). (right) Projection of the structure in the $b$-c plane. Lower parts of the figure show the $\mathrm{SbO}_{3}$ polyhedral units and the two separated chain-like rods within each unit cell extended along the $c$ axis can be observed ( $\mathrm{LP}$ configuration below $2 \mathrm{GPa}$ ). Upper parts of the figure show the $\mathrm{SbO}_{5}$ polyhedral units so that each rod gets linked with its four neighbor rods (enhanced bonds in the HP configuration above $2 \mathrm{GPa}$ ).

Along each rod, each $\mathrm{Sb}$ atom is mainly linked to three $\mathrm{O}$ atoms forming strong $\mathrm{Sb}-\mathrm{O}$ bonds of ca. $2.0 \AA$ (see bottom of Fig. 1); thus, $\mathrm{Sb}$ is threefold coordinated at room pressure. As regards the $\mathrm{O}$ coordination, the central $\mathrm{O}$ of each $\mathrm{Sb}_{2} \mathrm{O}_{3}$ unit along the chain, hereafter named $\mathrm{O} 1$, is bonded to the two $\mathrm{Sb}$ atoms of the same unit (bond distance of $2.0 \AA$ ). Besides, it must be noted that 01 is also weakly bonded to the two Sb atoms (bond distance of $2.6 \AA$ ) of the neighbour $\mathrm{Sb}_{2} \mathrm{O}_{3}$ unit along the infinite chain (see oblique dashed 
lines in the top right part of Fig. 1). On the other hand, each of the external $\mathrm{O}$ atoms of each $\mathrm{Sb}_{2} \mathrm{O}_{3}$ unit along the chain, hereafter named $\mathrm{O} 2$, is bonded to a $\mathrm{Sb}$ atom of the same unit (bond distance of $1.98 \AA$ ) and to one $\mathrm{Sb}$ atom of the neighbour $\mathrm{Sb}_{2} \mathrm{O}_{3}$ unit (bond distance of $2.02 \AA$ ). Additionally, each $\mathrm{O} 2$ atom is weakly bonded (bond distance of $2.5 \AA$ ) to a $\mathrm{Sb}$ atom in a neighbour chain (see almost vertical dashed lines in the top part of Fig. 1). This weak bond provides the link between the different chains or rods and leading to the formation of the 3D structure of valentinite. In summary, by taking these weak extra bonds into account, the structure of valentinite could be interpreted as formed by rods or needles with SbO5E units, where the coordination of $\mathrm{Sb}$ is 3 (with 2 extra-long bonds) and the coordination of $\mathrm{O} 1$ and $\mathrm{O} 2$ atoms is 2 (with 2 extralong bonds) and 2 (with 1 extra-long bond), respectively (see the polyhedral $\mathrm{SbO}_{5} E$ unit at the top part of Fig. 1).
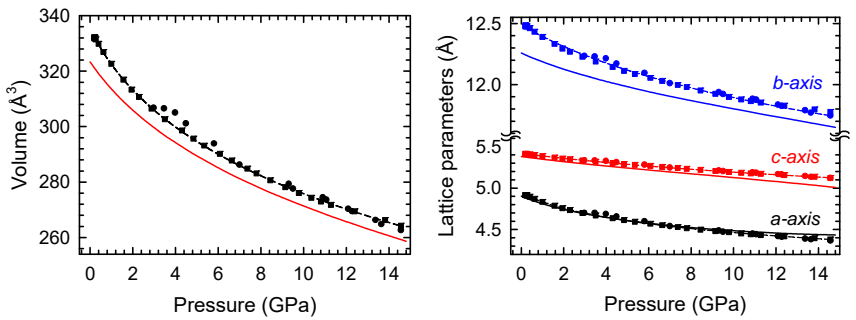

Figure 2. Pressure dependence of the experimental (symbols) and theoretical (solid lines) unit-cell volume (left) and lattice parameters (right) in $\beta$-Sb2O3. Filled squares and empty circles correspond to data of experiment 1 and experiment 2, respectively. The 3rd order BM EoS fit of experimental volume data (dashed line) is also shown. Experimental data fit of lattice parameters to the modified Murnaghan's EoS (dashed lines) are also shown.

Two angle-dispersive powder HP-XRD measurements of $\beta-\mathrm{Sb}_{2} \mathrm{O}_{3}$ at room temperature were conducted with a different pressuretransmitting medium (PTM). In experiment 1 (2), a 16:3:1 methanolethanol-water (MEW) mixture (helium) was used. New reflections, not corresponding to the LP phase of valentinite, were only observed above $15 \mathrm{GPa}$ in both experiments (see Fig. S2 and Fig. S3). This result suggests that quasi-hydrostatic conditions were maintained up to 15 $\mathrm{GPa}$ in both experiments and points to a phase transition above this pressure, in good agreement with the literature [35-37]. The phase transition is more evident in the case of enhanced quasi-hydrostatic conditions (experiment 2) than in the case of a quasi-hydrostatic environment (experiment 1). A pure HP phase was recorded above $16 \mathrm{GPa}$ in experiment 2, while experiment 1 revealed the coexistence of LP and HP phases from $16 \mathrm{GPa}$ up to $25 \mathrm{GPa}$. The analysis of the HP phase will be discussed below.

Le Bail analysis (partial Rietveld refinement) of powder XRD patterns of the LP phase of valentinite in experiment 1 (experiment 2) were conducted up to $14.6 \mathrm{GPa}$. For the refinement, the atomic coordinates of oxygen were fixed to those obtained by ab initio simulations in order to get more accuracy in the determination of the coordinates of $\mathrm{Sb}$ atoms. An example of the $2 \mathrm{D}$ diffraction pattern of experiment 2 and details of partial Rietveld refinement at selected pressures are shown in Fig. S5 and Table S1.

The analysis of the LP phase of $\beta-\mathrm{Sb}_{2} \mathrm{O}_{3}$ shows a smooth behaviour in the pressure dependence of the unit-cell volume up to $14.6 \mathrm{GPa}$ (Fig. 2 (left)), without any jump in volume (associated to a 1st order phase transition) or any apparent change in compressibility (associated to a $2^{\text {nd }}$ order phase transition). In fact, the data were easily fitted to a 3rd order Birch-Murnaghan (BM) equation of state (EOS) using EOSFIT software $[\mathbf{4 2 , 4 3 ]}$. Interestingly, the unit-cell volumes obtained at similar pressures in both experiments using MEW and helium are almost identical. This result contrasts with the large volume difference observed in arsenolite $\left(\mathrm{c}-\mathrm{As}_{2} \mathrm{O}_{3}\right)$ at similar pressures with these two PTMs due to a pressure-induced helium trapping in this molecular solid [10].

The bulk modulus obtained for $\beta-\mathrm{Sb}_{2} \mathrm{O}_{3}$ in experiment $1(2)$ is $\mathrm{B}_{0}=24(2)$ $\mathrm{GPa}\left(\mathrm{B}_{0}=27.4(8) \mathrm{GPa}\right)$. These values are in good agreement with our theoretical simulations $\left(\mathrm{B}_{0}=30.9(5) \mathrm{GPa}\right)$. The difference between both experiments is likely due to the lack of experimental data below $3 \mathrm{GPa}$ in experiment 2 (due to the helium loading procedure). The agreement between experiments and calculations supports the validity of our theoretical calculations performed under the GGAPBEsol approximation and will allow us to extend our analysis to different intrinsic properties of this compound. Such a point is crucial since we will show below that there are several IPTs of order higher than 2.

The HP dependence of the lattice parameters of $\beta-\mathrm{Sb}_{2} \mathrm{O}_{3}$ up to 14.6 GPa (Fig. 2(right)) exhibits a monotonic sublinear behaviour. Both experimental and theoretical data can be fitted to a modified $2^{\text {nd }}$ order BM EoS (see axial compressibilities and bulk moduli at room pressure in Table S3). The similarity of data from both experiments suggest that quasi-hydrostatic conditions are well maintained below 14.6 GPa in both XRD measurements. Consequently, the slight differences between the lattice parameters obtained at LPs for both experiments are likely due to an overpressure of the $\mathrm{Cu}$ signal in experiment 2 (range between 4 and $6 \mathrm{GPa}$ ) since the $\mathrm{Cu}$ powder was too close to the gasket. Note that the ratio between the different lattice parameters (Fig. S6) exhibits a monotonic variation without any indication of a possible $2^{\text {nd }}$ order IPT up to $14.6 \mathrm{GPa}$, unlike its counterpart $\beta-\mathrm{Bi}_{2} \mathrm{O}_{3} \cdot[\mathbf{1 8 , 4 4}]$

The c-axis shows the lowest axial compressibility at room pressure because its pressure behaviour is dominated by the longitudinal compressibility of the rods, mainly related to short $\mathrm{Sb}-\mathrm{O} 2$ bond distances inside the rods that link different $\mathrm{Sb}_{2} \mathrm{O}_{3}$ units along the caxis. We can also relate the compressibility of the $\mathrm{c}$-axis to the lateral compressibility of the $\mathrm{SbO}_{3} E$ or $\mathrm{SbO}_{5} E$ polyhedral units. The a-axis exhibits the highest compressibility because its change is dominated by the compression of the voids between rods; i.e. the compression of the cationic LEPs mainly oriented along the a-axis. In any case, a small contribution of the transversal compression of the relatively rigid rods along the a-axis cannot be neglected due to the bending of the long $\mathrm{Sb}-\mathrm{O} 2$ bonds that link the rods mainly directed along the $b$ axis (see almost vertical dashed lines in Fig. 1). Finally, the b-axis shows an intermediate compressibility mainly given by the compression of the long $\mathrm{Sb}-\mathrm{O} 2$ bonds and in a smaller extent to the transversal compression of the rods related to the longitudinal compressibility of $\mathrm{SbO}_{3} E$ (or $\mathrm{SbO}_{5} E$ ) units along the b-axis. Note that the $b$-axis compressibility is not affected by the compression of the short Sb-O1 bonds (Fig. S9). 
Fig. S7 shows the comparison between our theoretical and experimental (obtained from partial Rietveld refinement of HP-XRD patterns) $\mathrm{Sb}$ atomic coordinates. These results further confirm that we can rely on the HP evolution of the interatomic distances, polyhedral volume, and compressibilities of the different units obtained from our theoretical calculations. Interestingly, the theoretical pressure dependence of the atomic coordinates of $\mathrm{Sb}, \mathrm{O} 1$ and $\mathrm{O} 2$ atoms exhibit indications of the anomalous compressibility of valentinite. While some coordinates $\left(\mathrm{Sb}_{\mathrm{x}}, \mathrm{Sb}_{\mathrm{y}}\right.$ and $\left.\mathrm{O}_{\mathrm{z}}\right)$ show a monotonic trend with compression, the rest of free atomic coordinates, especially those corresponding to external $\mathrm{O} 2$ atoms, show a rather different behaviour depending on the pressure range. In particular, the coordinates of $\mathrm{O} 2$ reveal three different pressure ranges: i) from 0 to $4 \mathrm{GPa}$, ii) from 4 to $10 \mathrm{GPa}$ and iii) from 10 to 15 $\mathrm{GPa}$. In addition, the coordinate $\mathrm{Sb}_{\mathrm{z}}$ remains almost constant up to 2 $\mathrm{GPa}$ and then increases up to $10 \mathrm{GPa}$, where another slight change of tendency is observed. In summary, the atomic coordinates of compressed valentinite indicate structural changes at 2, 4 and $10 \mathrm{GPa}$ that are not detected in the evolution of the unit-cell volume and lattice parameters.

To conclude this section, we will now discuss the nature of the HP phase observed in $\beta-\mathrm{Sb}_{2} \mathrm{O}_{3}$ above $14.6 \mathrm{GPa}$. At this pressure, new reflections appear at $10.8,12.2,13.2$, and 16 degrees that reflect displacive shifts of some crystallographic planes of the LP phase. We remark that the most intense reflections of the LP phase remain after the phase transition to the HP phase is finished. This result indicates that the HP phase may maintain a direct relation with the original LP phase. A tentative assignment of the HP phase of valentinite has been performed by comparison with probable structures. The best concordance was achieved with an orthorhombic phase (s.g. Pcc2, see Fig. S10). This structure was predicted to be dynamically stable in $\mathrm{As}_{2} \mathrm{O}_{3}$ [5] and is a translationengleiche subgroup of index 2 to s.g. Pccn of $\beta-\mathrm{Sb}_{2} \mathrm{O}_{3}$. In order to corroborate this assignment, we have performed single-crystal HP-XRD measurements of mineral valentinite and $a b$ initio theoretical simulations of the proposed HP phase.

The structure of $\beta-\mathrm{Sb}_{2} \mathrm{O}_{3}$ at ambient conditions, with voids extending along the (001) direction provides the compound with a laminar character and a tendency to exfoliate along the (100) plane. This tendency to exfoliate would be avoided if we could get a perfectly oriented (100) single crystal; however, the mineral nature of this sample makes very difficult to obtain a pure (100) $\beta-\mathrm{Sb}_{2} \mathrm{O}_{3}$ single crystal, increasing the probability to form deformations in its crystalline structure. Under compression, the reflections at high $2 \theta$ angles quickly fade probably as a result of the slide of planes in our single crystalline sample. This fact produces a huge increase of the internal reflections $R_{\text {int }}$ value, which is of $\sim 30 \%$ at $16 \mathrm{GPa}$, thus preventing from a complete structural determination of the HP phase of $\beta-\mathrm{Sb}_{2} \mathrm{O}_{3}$. Despite the difficulty to solve the structure at 16 $\mathrm{GPa}$, we observe that the new structure also presents an orthorhombic symmetry, $a=4.590(14) \AA, b=11.113(7) \AA$ and $c=$ $4.9730(16) \AA$, very similar to that of the LP phase. The intensities and new reflections that emerge above $16 \mathrm{GPa}$ in the powder XRD experiment cannot be explained with s.g. Pccn. However, they are readily indexed in the non-centrosymmetric s.g. Pcc2. Both phases can be distinguished by systematic extinctions related to their symmetry elements. In particular, certain conditions for systematic extinctions ( $h 00, I=2 n ; 0 k 0, k=2 n ; h k 0, h+k=2 n$ ) do not apply in s.g. Pcc2. Unfortunately, due to the orientation of our crystal the $h 00$ reflections are not accessible. Furthermore, according to the powder diffraction pattern at $19.3 \mathrm{GPa}$ (Fig. S10), the $0 k 0$ and the $h k 0$ reflections are extremely weak hindering their localization in our single-crystal patterns (Fig. S11). Despite these limitations, the integration of reflections from single-crystal XRD measurements using the CrysAlis program [44] indicates that data are compatible with s.g. Pcc2.

The strongest support to the structural determination of the HP phase comes from single-crystal data. Even though the internal $R_{\text {int }}$ value is above $30 \%$, SHELXT [46] directly displays two sites for the $\mathrm{Sb}$ atom conforming a cationic structure almost identical to that of the LP structure and in perfect agreement with the structural determination from powder XRD. After several refinement iterations of the cationic structure with program SHELXL [47], the R1 value goes down to a minimum of $35 \%$, but it is not reduced when we introduce the $O$ positions (Table S2). Such a drawback, which is the result of the poor $R_{\text {int }}$ value prevents any total structural determination but confirms the structural determination made by powder HP-XRD measurements. The two atoms ( $\mathrm{Sb}$ and $\mathrm{O} 2$ ) that are in $8 e$ crystallographic sites in the LP phase split into two crystallographic sites each with $4 e$ symmetry in the HP phase and the atom (01) that is in the $4 d$ Wyckoff site in the LP phase splits into a $2 b$ and a $2 c$ crystallographic site in the HP phase. The determination of four out of the six positions of the HP phase with single-crystal XRD is further validated by our ab initio theoretical simulations (Table 2) that confirm the orthorhombic HP phase of $\beta-\mathrm{Sb}_{2} \mathrm{O}_{3}$ as a distortion of its LP phase instead of the monoclinic structure proposed by Zou et al. [37]

\section{Vibrational properties of $\beta-\mathrm{Sb}_{2} \mathrm{O}_{3}$ under compression}

Raman and infrared (IR) scattering spectroscopies are very sensitive tools, much local than XRD measurements, that can show changes in the configuration of interatomic bonds. Consequently, they might reflect the anomalous compressibility of the LP phase of valentinite already commented. According to group theory, the centrosymmetric orthorhombic phase of $\beta-\mathrm{Sb}_{2} \mathrm{O}_{3}$, with $\mathrm{D}_{2 \mathrm{~h}}(\mathrm{mmm})$ point group, should have 60 zone-centre phonon modes belonging to the following irreducible representation: $\Gamma=7 A_{g}+7 A_{u}+7 B_{1 g}+7 B_{1 u}$ $+8 B_{2 g}+8 B_{2 u}+8 B_{3 g}+8 B_{3 u}$. [48] Three of these modes $\left(B_{1 u}, B_{2 u}\right.$, and $\left.B_{3 u}\right)$ correspond to acoustic phonons, while the seven $A u$ modes are silent. This results in 30 Raman-active (gerade) optical modes $\left(\Gamma_{R}=\right.$ $7 \mathrm{~A}_{\mathrm{g}}+7 \mathrm{~B}_{1 \mathrm{~g}}+8 \mathrm{~B}_{2 \mathrm{~g}}+8 \mathrm{~B}_{3 \mathrm{~g}}$ ) and 20 IR-active (ungerade) optical modes $\left(\Gamma_{1 R}=6 B_{1 u}+7 B_{2 u}+7 B_{3 u}\right)$. 

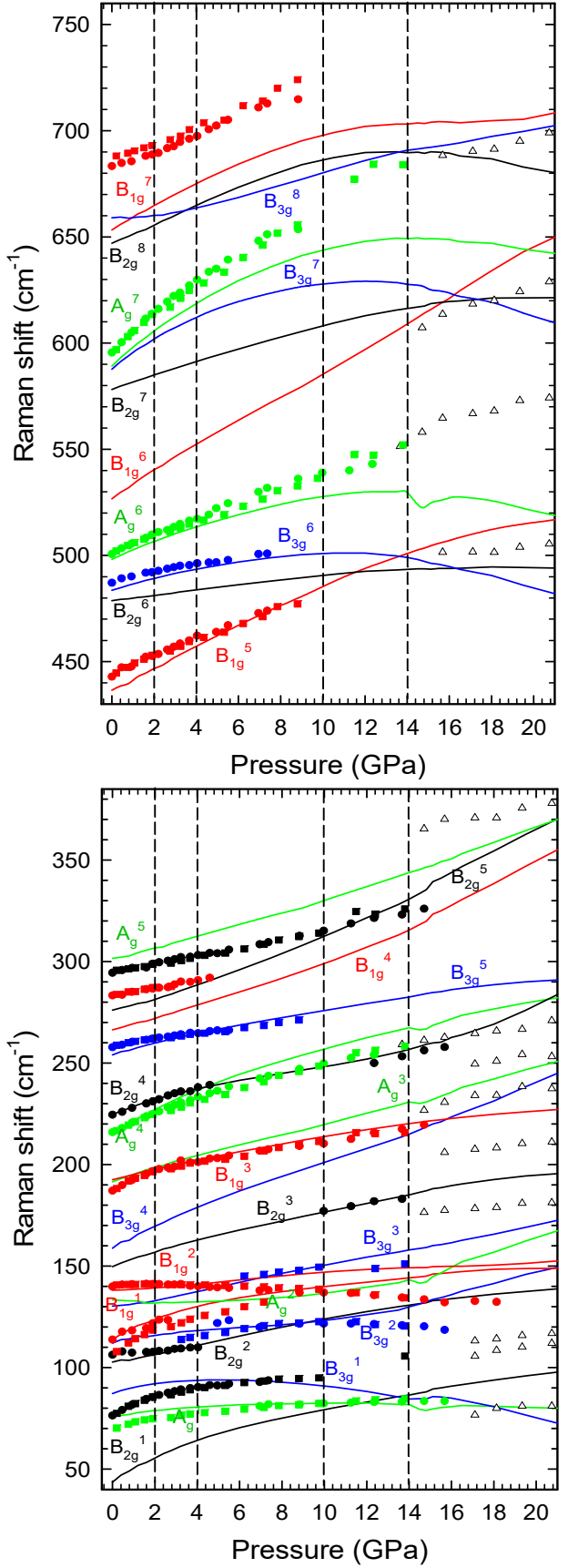

Figure 3. Pressure dependence of the theoretical (lines) and experimental Raman-active mode frequencies of $\beta-\mathrm{Sb}_{2} \mathrm{O}_{3}$ obtained from powder (circles) and single crystals (squares) below $400 \mathrm{~cm}^{-1}$ (bottom) and above $400 \mathrm{~cm}^{-1}$ (top). Each colour corresponds to an assigned symmetry: green $\left(A_{g}\right)$, red $\left(B_{1 g}\right)$, black $\left(B_{2 g}\right)$ and blue $\left(B_{3 g}\right)$, whose labels are situated near the theoretical curves. The experimental Raman frequencies of the HP phase observed above $15 \mathrm{GPa}$ (empty triangles) are also shown.

The RS spectrum of $\beta-\mathrm{Sb}_{2} \mathrm{O}_{3}$ at room pressure is well known $[35,36,49-52]$ and has been traditionally divided into two different regions separated by a phonon gap: i) the low-frequency region (from 50 to $400 \mathrm{~cm}^{-1}$ ) and ii) the high-frequency region (from 400 to $\left.800 \mathrm{~cm}^{-1}\right)$. The most intense peaks are located in the low-frequency region that is dominated by a peak near $140 \mathrm{~cm}^{-1}$. Vibrational modes of $\beta-\mathrm{Sb}_{2} \mathrm{O}_{3}$ above (below) $400 \mathrm{~cm}^{-1}$ were assigned to internal (external) vibrational modes of pyramidal $\mathrm{SbO}_{3}\left(\right.$ or $\left.\mathrm{SbO}_{3} E\right)$ molecules by many authors. On this basis, Cody et al. [49] suggested that internal modes should be similar in the different crystalline structures of $\mathrm{Sb}_{2} \mathrm{O}_{3}$, senarmontite and valentinite; while external modes should depend on the particular space group of the crystalline structure due to the different arrangement of molecules inside the unit cell of both compounds [49]. Later, Miller and Cody used the molecule $\mathrm{O}_{2} \mathrm{Sb}-\mathrm{O}-\mathrm{SbO}_{2}$ to describe the internal modes of vitreous $\mathrm{Sb}_{2} \mathrm{O}_{3}$ and assigned internal vibrational modes of this molecule [47]. Finally, a better approach to interpret the RS and IR spectra of valentinite has been performed by Voit et al., who used $\mathrm{Sb}_{10} \mathrm{O}_{15}$ units to model the vibrational spectra [49]. Our calculations do not support the separation of vibrational modes of valentinite into external and internal modes of the $\mathrm{SbO}_{3}$ molecules, as suggested by the previous works, since all molecules are linked together in a complex double chain structure which forms infinite $\left(\mathrm{Sb}_{2} \mathrm{O}_{3}\right)_{\infty}$ chain molecules. Details of the vibrational modes of valentinite and its associated atomic motions are provided in Figs. S11-S13 and S18S22.

A classification of the vibrational spectrum of valentinite is provided by the theoretical phonon density of states (Fig. S12), which allows dividing it into three main ranges: i) low-frequency (below $100 \mathrm{~cm}^{-1}$ ), where the vibrations are dominated by the motion of the Sb cations; ii) middle-frequency $\left(100-300 \mathrm{~cm}^{-1}\right)$, where the vibrations are associated to the motion between polyhedral $\mathrm{SbO}_{3}$ units as a group (both $\mathrm{Sb}$ and $\mathrm{O}$ atoms move with predominance of $\mathrm{Sb}$ movement below $230 \mathrm{~cm}^{-1}$ and of $O$ motion above $230 \mathrm{~cm}^{-1}$ ); and iii) highfrequency (above $300 \mathrm{~cm}^{-1}$ ), where there are different kinds of mixed bending and stretching vibrations of $\mathrm{SbO}_{3}$ units which mainly involve motion of oxygen atoms.

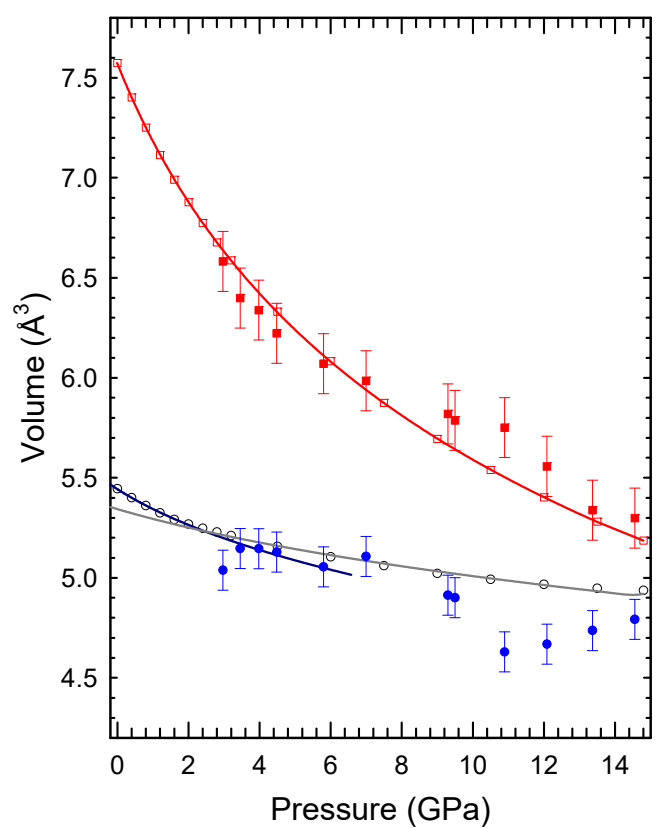

Figure 4. Pressure dependence of the experimental (solid symbols) and theoretical (empty symbols) volume of the $\mathrm{SbO}_{5} \mathrm{E}$ polyhedral unit (blue) and the empty cavity (red) in $\beta-\mathrm{Sb}_{2} \mathrm{O}_{3}$. The $3^{\text {rd }}$ order BM EoS fits (solid lines) of theoretical data are also shown. 

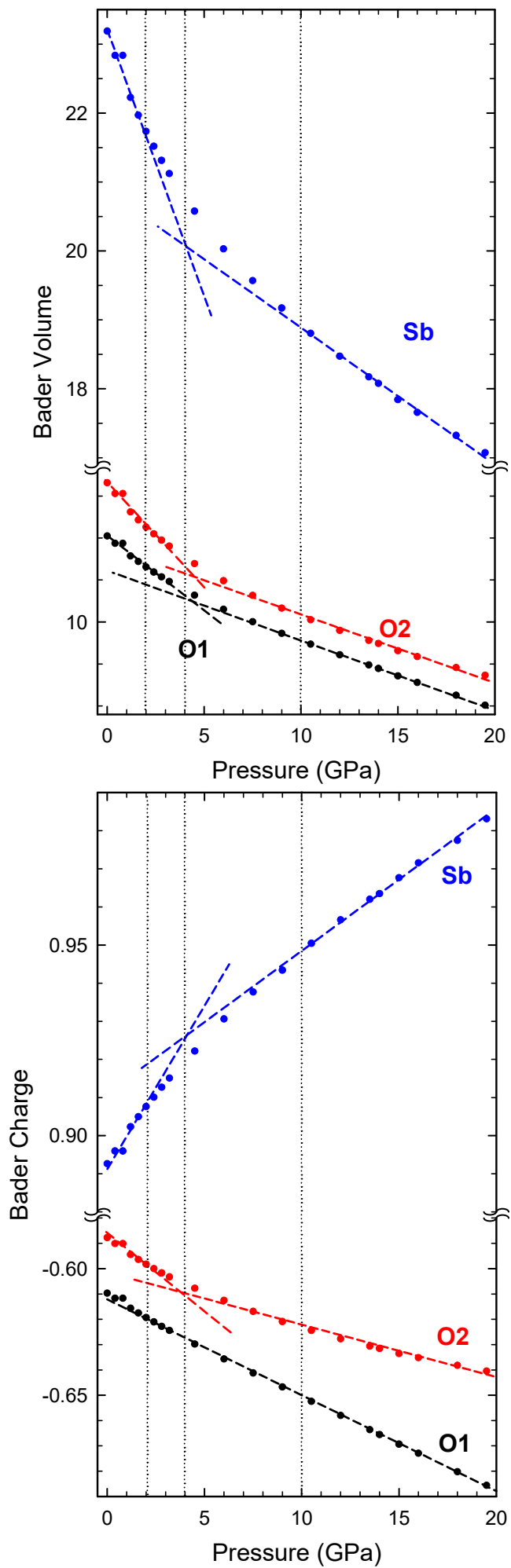

Figure 5. Evolution of the theoretical Bader volumes (top) and charges (bottom) of the non-equivalent atoms in $\beta-\mathrm{Sb}_{2} \mathrm{O}_{3}$. Dashed straight lines are guides to the eyes in order to help visualize the different tendencies observed.

Unpolarized HP-RS measurements of $\beta-\mathrm{Sb}_{2} \mathrm{O}_{3}$ were performed up to 20.7 GPa in both synthetic powders and single crystals of mineral valentinite (see Fig. S16 and Fig. S17). The RS spectra of valentinite at different pressures are in good agreement with previous reports

[35,36,49-52]. New Raman-active modes are observed above 14 $\mathrm{GPa}$, thus confirming the transition to the HP phase already found by XRD measurements. Unfortunately, the low quality of the RS data above $14 \mathrm{GPa}$, even from single-crystal data, does not help to get an unambiguous assignment of the HP phase of $\mathrm{\beta}_{-} \mathrm{Sb}_{2} \mathrm{O}_{3}$.

The pressure dependence of the experimental and theoretical Raman-active modes of the LP phase in valentinite (Fig. 3 ) shows a rather good agreement between all data, especially above $150 \mathrm{~cm}^{-1}$ where Raman-active modes are more separated. The comparison of theoretical and experimental frequencies and pressure coefficients of the Raman-active modes in valentinite (see Tables S3 and S4) has allowed us to assign in a tentative way the symmetry of many observed Raman peaks. Below $150 \mathrm{~cm}^{-1}$, the symmetry assignment of the Raman-active modes is doubtful for most modes due to the overlapping of many theoretical modes. The pressure dependence of the theoretical IR-active and silent mode frequencies is shown in Fig. S18 and their frequencies and pressure coefficients are summarized in Table S6. The most striking feature of the pressure dependence of the vibrational modes of valentinite is that many modes show a strong non-linear pressure dependence showing changes around 2, 4 and $10 \mathrm{GPa}$. These changes agree with those observed in the free atomic coordinates of the Wyckoff positions, as already commented.

\section{Discussion}

A thorough analysis of the experimental and theoretical volumes of the $\mathrm{SbO}_{5} E$ polyhedral units and of the empty cavities of the LP phase of $\beta-\mathrm{Sb}_{2} \mathrm{O}_{3}$ under compression (Fig. 4) shows that the bulk compressibility of valentinite is strongly dominated by the behaviour of its structural voids. The compression of the theoretical empty cavity volume shows a monotonic variation up to $14.6 \mathrm{GPa}$ that fits perfectly to a single $3^{\text {rd }}$ order $\mathrm{BM} \operatorname{EoS}\left(\mathrm{B}_{0}=16.9(2) \mathrm{GPa}\right)$. On the contrary, a fit of the theoretical $\mathrm{SbO}_{5} E$ polyhedron volume as a function of pressure to a single $3^{\text {rd }}$ order BM EoS in the same pressure range is not possible, thus revealing the existence of two different compressibility regions below and above $2 \mathrm{GPa}$. Fit to a $3^{\text {rd }}$ order $\mathrm{BM}$ EoS below (above) 2 GPa yields $B_{0}=48(5) \mathrm{GPa}\left(\mathrm{B}_{0}=102(10) \mathrm{GPa}\right)$. Given the existence of $2^{\text {nd }}$ order IPTs in other sesquioxides, like $\beta-\mathrm{Bi}_{2} \mathrm{O}_{3}$, we wonder whether the compressibility changes in the $\mathrm{SbO}_{5} E$ polyhedron at $2 \mathrm{GPa}$ could be considered as hidden or internal $2^{\text {nd }}$ order IPT. These adjectives indicate that this transition is not reflected in the bulk behaviour, already analysed, because it remains hidden by the strong compressibility of the linear empty cavity that dominates the unit-cell volume compressibility of valentinite.

The $F-f$ plot, being $F$ the reduced pressure and $f$ the Eulerian strain, is a more sensitive tool than the unit-cell volume vs. pressure plot to observe slight changes in the compressibility $[\mathbf{5 2}, \mathbf{5 3}]$. Therefore, we have analysed the $F-f$ plot for the theoretically simulated bulk structure, polyhedral $\mathrm{SbO}_{5} E$ unit, and the empty cavity under compression (Fig. S8) in order to get a better illustration of the different compressibility ranges of valentinite. The $F-f$ plots have unveiled more than a critical pressure value along the stability pressure range of valentinite. The $F-f$ plot of the bulk structure reveals a compressibility change around $10 \mathrm{GPa}$ that is observed also 
in the polyhedral unit and the empty cavity. On the other hand, the $F$ - $f$ plot of the polyhedral unit confirms a compressibility change at 2 GPa. Finally, it must be noted that the $F-f$ plot of the empty cavity indicates an additional change around $4 \mathrm{GPa}$. These pressure values agree with those already found in our study of the lattice dynamics and of the theoretical dependence of the free atomic coordinates.

Complementary information about the different compressibility regions of valentinite is provided by the HP behaviour of the $\mathrm{Sb}-\mathrm{O}$ interatomic distances inside the $\mathrm{SbO}_{5} E$ polyhedral units (Fig. S8). A strong decrease of the theoretical long $\mathrm{Sb}-\mathrm{O} 2$ distance (theoretically around $2.42 \AA$ and experimentally around $2.5 \AA$ at $0 \mathrm{GPa}$ ) is observed in the range from 0 to $4 \mathrm{GPa}$. Additionally, there is a change in the sign of the slope of the short $\mathrm{Sb}-\mathrm{O} 1$ bond in the same pressure range. Note that this is the bond from the central $\mathrm{O} 1$ atom to the two $\mathrm{Sb}$ atoms of the same $\mathrm{Sb}_{2} \mathrm{O}_{3}$ molecule. The changes in $\mathrm{Sb}-\mathrm{O}$ distances indicate that the main effect of pressure up to 2-4 GPa is to bring neighbour rods closer; while above this pressure range, pressure mainly tends to increase the packing inside the rods by compressing the atoms inside the $\mathrm{SbO}_{3}$ molecular units.

Additional evidence of the extraordinary changes observed at 2, 4, and $10 \mathrm{GPa}$ in valentinite are provided by a detailed comparison of the RS measurements (in single crystals and powders) and theoretical lattice dynamics calculations (Figs. 3, S15 and S16). At LPs, most experimental Raman-active frequencies show a blueshift with increasing pressure. This is also the case of the most intense Raman peak, located near $140 \mathrm{~cm}^{-1}$ at room pressure and tentatively attributed to the $\mathrm{B}_{1 \mathrm{~g}}{ }^{2}$ mode (Fig. S19). However, this mode shows a negative pressure coefficient above $2 \mathrm{GPa}$ (Fig. S18), which agrees with previous HP-RS measurements $[35,36]$. In fact, changes in the pressure coefficients of vibrational frequencies around $2 \mathrm{GPa}$ are also observed in other theoretical Raman-active modes (Fig. 3) as well as in IR-active and silent modes (Fig. S18). In particular, the $\mathrm{Ag}_{\mathrm{g}}{ }^{2}$ and $\mathrm{B}_{3 \mathrm{~g}}{ }^{8}$ modes show negative (positive) pressure coefficient in $\beta-\mathrm{Sb}_{2} \mathrm{O}_{3}$ below (above) $2 \mathrm{GPa}$.

Changes in slope of vibrational frequencies around $4 \mathrm{GPa}$ are experimentally evidenced by the experimental mode near $225 \mathrm{~cm}^{-1}$, assigned to the $\mathrm{B}_{2 \mathrm{~g}}{ }^{4}$ mode. Note that this mode has a smaller pressure coefficient than the adjacent $\mathrm{Ag}_{\mathrm{g}}{ }^{4}$ mode so both modes overlap above $5 \mathrm{GPa}$ and become separated again above $12 \mathrm{GPa}$ (see detail in Fig. S26). Similarly, the theoretical $\mathrm{B}_{3 \mathrm{~g}}{ }^{1}\left(\mathrm{~A}_{\mathrm{g}}{ }^{2}\right)$ mode shows positive (negative) pressure coefficient below (above) $4 \mathrm{GPa}$, and the converse above this pressure.

Finally, it must be stressed that many Raman-active modes $\left(A_{g}{ }^{1}, B_{2 g}{ }^{4}\right.$, $\left.\mathrm{B}_{3 \mathrm{~g}}{ }^{6}, \mathrm{~B}_{3 \mathrm{~g}}{ }^{7}, \mathrm{~B}_{2 \mathrm{~g}}{ }^{8}, \mathrm{~B}_{1 \mathrm{~g}}{ }^{7}\right)$ and IR-active and silent modes show a change in slope at $10 \mathrm{GPa}$. In particular, most of these modes show a softening of their frequencies above this pressure, likely related to the increase of the short $\mathrm{Sb}-\mathrm{O} 2$ bond distance (Fig. S9). This behaviour suggests the existence of a general instability of the LP phase of $\beta-\mathrm{Sb}_{2} \mathrm{O}_{3}$ above $10 \mathrm{GPa}$ (see also the strange kink in theoretical frequencies near 15 $\mathrm{GPa}$ ). In this regard, we must recall that a complete softening (frequency going down to zero) of a vibrational mode is a signature of a "real" $2^{\text {nd }}$ order phase transition according to Landau theory of phase transitions, as it occurs in $\beta-\mathrm{Sb}_{2} \mathrm{O}_{3}$ and $\beta-\mathrm{Bi}_{2} \mathrm{O}_{3}[19,44]$. In this context, the calculated phonon dispersion curves of $\beta-\mathrm{Sb}_{2} \mathrm{O}_{3}$ (Fig.
S28) indicate that there is no complete softening of any vibrational mode along the Brillouin zone in the whole range up to $15 \mathrm{GPa}$. This result implies that we cannot consider the changes observed in the compressibility of valentinite at 2, 4 and $10 \mathrm{GPa}$ as belonging to a $2^{\text {nd }}$ order IPT, so they must be ascribed to IPTs of order higher than 2 .

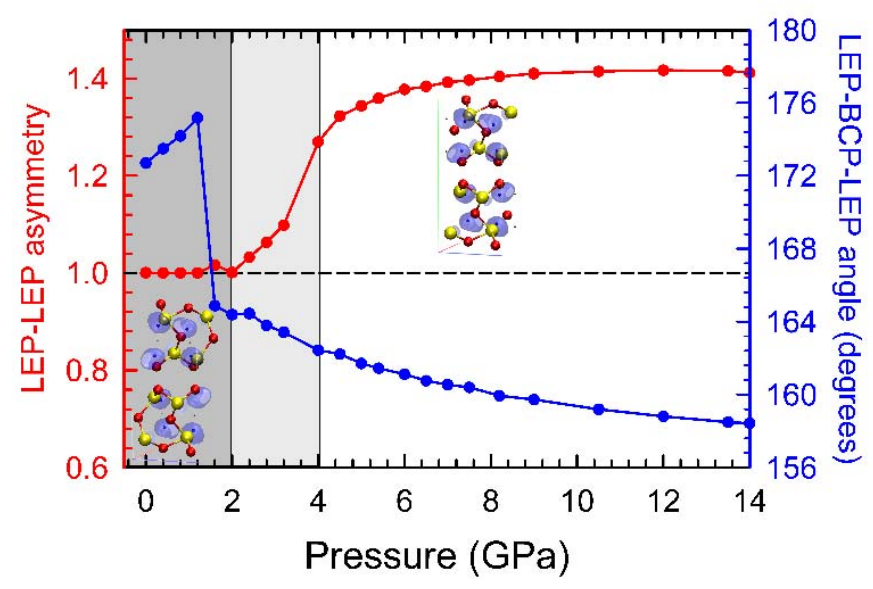

Figure 6. Evolution of the LEP-LEP asymmetry (red) and the angle formed by neighboring LEPs and the BCP (blue) under compression. The location of the BCP in the structure is displayed in the inset of the figure for each pressure range proposed.

To conclude the discussion regarding the vibrational properties of valentinite under compression, we must mention that the changes in compressibility at different pressures have been confirmed not only by changes in slopes of the frequencies of vibrational modes, but also by the accurate analysis of the pressure dependence of the full width half-maximum (FWHM) of several high-intensity Raman-active modes, as obtained from HP-RS measurements in single-crystal samples (Fig. S27). The HP evolution of the FWHM in two experimental Raman-active modes (one in the low-frequency region and another in the high-frequency region) show that changes in their FWHM occur at roughly the same critical pressures where changes in the F-f plots are observed. In summary, all HP-RS results give experimental support to the conclusions obtained by the analysis of the theoretical simulations regarding the existence of hidden or internal structural changes in valentinite around 2, 4, and $10 \mathrm{GPa}$. In order to find additional evidence for the different ranges of compressibility of valentinite, we have studied the evolution of the topology of the electronic charge distribution by analyzing the atomic Bader volumes and charges in $\beta-\mathrm{Sb}_{2} \mathrm{O}_{3}$ (Fig. 5). According to Bader's theory, the change in the topological distribution of the charge around an atom may reveal a variation of the molecular geometry since their electronic distribution should be invariant to pressure [53]. The Sb volume and charge reveal a change of tendency above 2 and $10 \mathrm{GPa}$ that agrees roughly with the pressure where the compressibility of the $\mathrm{SbO}_{5} E$ polyhedral volume changes. The $\mathrm{O} 2$ volume and charge show changes in tendency between 2 and $4 \mathrm{GPa}$, which are complementary to that observed in Sb. Finally, the 01 volume and charge follow almost linear trends with a small change of trend near $2 \mathrm{GPa}$. These results are consistent with the 01 central position in the rod and suggest that $\mathrm{O} 1$ atoms are barely involved in 
the compressibility changes observed in valentinite. Consequently, these results show that the anomalous compressibility of the polyhedral $\mathrm{SbO}_{5} E$ unit around 2-4 GPa may be related to a charge transfer between $\mathrm{Sb}$ and $\mathrm{O} 2$ atoms. This is a clear indication that the different rods start to interact in a more intense way above this pressure range. Thus, we can consider that above this pressure range the coordination of the $\mathrm{Sb}$ atom reaches a real $3+1$ coordination with a loss of the isolated rod character at LP; i.e. a quasi-1D to a 3D transition occurs between 2 and $4 \mathrm{GPa}$ in valentinite. Therefore, changes between 2 and $4 \mathrm{GPa}$ could be considered to be related to internal or hidden $2^{\text {nd }}$ order IPTs not reflected in the bulk compressibility of $\beta-\mathrm{Sb}_{2} \mathrm{O}_{3}$. Noteworthy, a $2^{\text {nd }}$ order IPT has also been found in $\beta-\mathrm{Sb}_{2} \mathrm{O}_{3}[19,44]$ and also in $\mathrm{Sb}_{2} \mathrm{Te}_{3}[54]$ and $\mathrm{BiMn}_{2} \mathrm{O}_{5}$ [55] where the $2^{\text {nd }}$ order IPT was also revealed by the analysis of the evolution of the Bader charge under compression.

Another evidence of the occurrence of subtle compressibility changes in valentinite under compression comes from the analysis of the pressure dependence of the orientation and distortion of the cationic LEPs, which has been studied by the topology of the electronic localization function (ELF). The pressure dependence of the LEP orientation, given by the angle formed by the interacting LEPs and its common bond critical point (BCP), reveals a drastic change at $2 \mathrm{GPa}$ (Fig. 6). The change in the orientation of the LEPs around $2 \mathrm{GPa}$ agrees nicely with the features observed in the analysis of the atomic Bader volumes and charges and with those observed in the structural and vibrational properties of valentinite. Therefore, we can conclude that the anomalous behaviour of valentinite at 2 $\mathrm{GPa}$ is caused by a sudden reorientation of the cationic LEPs that form the structural voids or channels in valentinite. At room pressure, LEPs are oriented towards the centre of the empty cavity so they interact with their neighbouring LEPs belonging to different $a-c$ crystallographic planes. The strong compression of the empty cavity along the a-axis (shown by the strong decrease of the inter-rod bond distances) shortens the distance between neighbour LEPs belonging to the same a-c crystallographic plane in such a way that these LEPs start to interact. This leads to an abrupt change in the LEPBCP-LEP angle near $2 \mathrm{GPa}$.

On the other hand, the LEP-LEP asymmetry, measuring the distortion of the two LEP-BCP distances (Fig. 6), indicates a negligible distance asymmetry between the two interacting LEPs below $2 \mathrm{GPa}$. This asymmetry increases above $2 \mathrm{GPa}$ up to around $4 \mathrm{GPa}$ when it becomes constant. This behaviour can be explained by the strong decrease of the voids volume and the strong reduction of the long interatomic Sb-O2 distances at LPs. Up to $2 \mathrm{GPa}$, the cation LEPs suffer a gradual reorientation remaining barely undistorted; the relative asymmetry with respect to the room pressure value is 1 (no asymmetry). Above $2 \mathrm{GPa}$, the strong compression between neighbour rods causes a sudden reorientation of the cationic LEPs and the effect of pressure begins to distort the cationic LEPs themselves. This distortion is reflected in the increase of the relative asymmetry of the LEP-BCP distances (deviation with respect to 1 that corresponds to the same LEP-BCP distance in the two interacting LEPs). This means that the distortion of the LEP cloud is larger for one LEP than for its neighbour one. The difference in the LEP distortions reaches a maximum near $4 \mathrm{GPa}$; i.e., the LEP cloud considered as an ellipsoid becomes more and more stretched up to $4 \mathrm{GPa}$. Above this pressure, the LEP cloud cannot be further compressed and the relative LEP asymmetry remains constant. This means that the effect of pressure above $4 \mathrm{GPa}$ is reflected on the decrease of the long interatomic $\mathrm{Sb}-\mathrm{O}$ distances and specially in the decrease the second shortest $\mathrm{Sb}-\mathrm{O} 2$ distance (Fig. S8). The compression of the internal Sb$\mathrm{O} 1$ bonds above $4 \mathrm{GPa}$ indicates that a real 3D behaviour is found above this pressure.

The anomalous hidden or internal behaviour at 2 and $4 \mathrm{GPa}$ cannot be interpreted as pure $2^{\text {nd }}$ order IPTs due to the lack of discontinuities in thermodynamic parameters, which avoids their assignment in the Ehrenfest's classification [56]. New and extended definitions of phase transitions given by $\mathrm{Ma}$ and Wang [57] introduces type-I transitions. Such a type of transitions only requires two stable states without variable discontinuity requirement and are called continuous transitions. Unfortunately, the definition of a different initial and final stable state is quite difficult to identify in our case. Another point is that by definition a phase transition implies changes in atomic bonds (coordination, symmetry or arrangement); however, to our knowledge, the changes of interaction between unpaired electrons, like LEPs, in inorganic solids under compression, has never been studied up to our knowledge. In valentinite, the different interactions between neighbour LEPs may cause subtle changes in the properties of valentinite. However, these changes cannot be assigned to a $2^{\text {nd }}$ order IPT in the Ehrenfest's classification and consequently should be attributed to a transition of order higher than 2.

Finally, we would like to remark that all F-f plots (Fig. S7) show a tendency change at $10 \mathrm{GPa}$, in perfect correlation with a general softening of many vibrational modes (Figs. 3 and S17). A response to compression not observed by the analysis of the electronic topology. As already commented, the general softening of Raman-active and IR-active modes observed above $10 \mathrm{GPa}$ may be the result of an ongoing instability, probably the onset of the phase transition experimentally observed above $15 \mathrm{GPa}$. However, our ab initio calculations do not show any complete softening of the theoretical vibrational modes up to $14 \mathrm{GPa}$. Note that the $\mathrm{B}_{2 \mathrm{u}}{ }^{1}$ mode near $25 \mathrm{~cm}$ ${ }^{1}$ at room pressure shows an almost complete freezing near $15 \mathrm{GPa}$. This indicates that the structure becomes fully unstable above 14 GPa when the transition occurs. For this reason, the existence of an electronic-driven IPT around $10 \mathrm{GPa}$ has also been considered. Our theoretical calculations of the electronic band structure at several pressures (Fig. S29) show a progressive decrease of the bandgap of valentinite. However, neither a change from semiconducting-tometallic nor a change in the topology of the Fermi level around 10 GPa has been observed. Consequently, the origin of the instability occurring above $10 \mathrm{GPa}$ in valentinite is still unknown and will deserve further studies. In any case, the theoretical interatomic distances in Fig. 59 allows an interesting reading. If we make the hypothesis that the $3+1$ coordination of Sb starts at $2 \mathrm{GPa}$ (when the large $\mathrm{Sb}-\mathrm{O} 2$ distance decreases below $2.36 \AA$ ) the increase in coordination to $3+1+1$ of $\mathrm{Sb}$ would start above $10 \mathrm{GPa}$ (when the much larger Sb-01 distance decreases below $2.38 \AA ̊$ ). Moreover, 
there is a change in the theoretical slope of the two shortest $\mathrm{Sb}-\mathrm{O} 2$ bond distances above $10 \mathrm{GPa}$ (Fig. S9). The shortest one exhibits a reduction of the slope while the largest one exhibits an increase of the slope; i.e. it enlarges at a higher rate what suggests a structural instability. Consequently, the increase of coordination of $\mathrm{Sb}$ above 10 $\mathrm{GPa}$, thanks to the interaction of the central parts of neighbour $\mathrm{Sb}_{2} \mathrm{O}_{3}$ units in the same rod (involving large Sb-O1 bonds), lead to unusual changes of the $\mathrm{Sb}-\mathrm{O} 2$ distance and could trigger the instability of the structure of valentinite. In the HP phase, the polyhedral unit reaches a fivefold coordination confirming the trend observed with compression. In summary, our analysis shows that the anomalous compressibility of valentinite at 2 and $4 \mathrm{GPa}$ is related to changes of the cationic LEPs that affect the connectivity of external $\mathrm{O} 2$ atoms, while that occurring at $10 \mathrm{GPa}$ is not related to cationic LEPs but likely related to changes in the connectivity of internal $\mathrm{O} 1$ atoms that in turn affect in a strange way the compressibility of $\mathrm{Sb}-\mathrm{O} 2$ bonds.

\section{Experimental}

\section{Experimental Details}

Synthetic $99.9 \%$ pure white powder of $\beta-\mathrm{Sb}_{2} \mathrm{O}_{3}$ samples used in the present experiments were acquired from Sigma-Aldrich Inc., while fully transparent single crystals of valentinite were obtained from Lac Nicolet Mine in Quebec (Canada). Angledispersive powder HP-XRD measurements at room temperature up to $25 \mathrm{GPa}$ were conducted in a membrane-type diamond anvil cell (DAC) at the BLO4-MSPD beamline of ALBA synchrotron in two different experiments. The sample was loaded inside DAC using either a MEW mixture (experiment 1 ) or helium (experiment 2) as a PTM. The pressure inside the cavity was calibrated through the EoS of copper [58]. Incident monochromatic beam with wavelength of $0.4246 \AA$ was focused to $20 \times 20 \mu \mathrm{m}^{2}$ and cleaned using a pinhole of $50 \mu \mathrm{m}$ [59]. Images covering a $2 \theta$ range up to $20^{\circ}$ were collected using a SX165 CCD located at $240 \mathrm{~mm}$ from sample. Integrated XRD patterns profiles as a function of $2 \theta$ were obtained with the Fit2D software [60]. Le Bail analysis (partial Rietveld refinement) of experimental diffraction patterns corresponding to experiment 1 (experiment 2) were carried out with GSAS package software [61,62]. Partial Rietveld refinement was performed by fitting the atomic coordinates of $\mathrm{Sb}$ and fixing the atomic coordinates of $\mathrm{O} 1$ and $\mathrm{O} 2$ to those obtained by ab-initio simulations corresponding to each pressure, in order to obtain a better fit of atomic coordinates of Sb atoms (see Fig. S7 and Table S1). This procedure was performed because lighter elements are more sensitive to errors in their determination by XRD measurements. Small variations of the free Wyckoff sites may lead to strong changes in interatomic distances, which can avoid an adequate precision for the experimental determination of bond lengths. The background of each XRD powder pattern has been fitted to a shifted Chebyshev function of $1^{\text {st }}$ kind and the peak shape has been analysed using the profile coefficients for Simpson's rule integration of pseudovoigt function implemented in GSAS package.

For the single crystal XRD experiment a (100)-oriented single crystal was loaded into a Merrill-Bassett diamond-anvil cell, equipped with
$500 \mu \mathrm{m}$ culet diamonds. The sample with a surface of $150 \times 40 \mu \mathrm{m}^{2}$ was loaded in the pressure chamber made in the $200 \mu \mathrm{m}$ hole of a tungsten gasket that had been preindented to $80 \mu \mathrm{m}$ in thickness. The pressure transmitting medium was $\mathrm{He}$ and a ruby chip was included for pressure determination. The XRD data were collected at the Beamline 119, at Diamond Light Source, using the Newport kappa-geometry 4-circle diffractometer fitted with a Dectris Pilatus $300 \mathrm{~K}$ pixel-array photon-counting detector. The dataset was collected using a wavelength of $\lambda=0.4895 \AA$, with a step size and exposure time of $0.2^{\circ}$ and 0.4 seconds respectively. The data were collected using a series of $\omega$ scans to give the optimal completeness when using a diamond anvil cell. The data were integrated with the program CrysAlisPro [45] which incorporates routines that omit regions of the detector shaded by the diamond-anvil cell from integration.

Room-temperature unpolarized HP-RS measurements on powder (up to $22 \mathrm{GPa}$ ) and single crystal $\beta-\mathrm{Sb}_{2} \mathrm{O}_{3}$ (up to $15 \mathrm{GPa}$ ) samples, using MEW as a PTM, were carried out with a Horiba Jobin Yvon LabRAM HR spectrometer equipped with a thermoelectrically cooled multichannel CCD detector that enables a spectral resolution better than $2 \mathrm{~cm}^{-1}$ [63]. RS measurements were excited using the $6328 \AA$ line of a He:Ne laser. Special attention was paid during the experimental measurements to avoid the undesired laser heating effects already observed in the cubic polymorph of the antimony oxide [64]. The ruby fluorescence scale [65] was used to determine the pressure. It must be noted that MEW and helium provide nearly hydrostatic conditions up to $10 \mathrm{GPa}$ and $20 \mathrm{GPa}$, respectively, and quasi-hydrostatic conditions up to the maximum pressure attained in our experiments $[66,67]$

\section{Theoretical Details}

$A b$-initio total-energy calculations were performed for $\beta-\mathrm{Sb}_{2} \mathrm{O}_{3}$ within the density functional theory (DFT) [68] using the plane-wave method and the pseudopotential theory with the Vienna Ab-initio Simulation Package (VASP) [69]. The projector-augmented wave scheme (PAW) [70-73] was employed and the basis set of plane waves was extended up to an energy cutoff of $520 \mathrm{eV}$ in order to achieve highly converged results and accurate description of the electronic properties. The exchange-correlation energy was taken in the generalized gradient approximation (GGA) with the PBEsol [74] prescription. To ensure a high convergence of 1-2 meV per formulaunit in the total energy as well as an accurate computation of the forces on the atoms, the integrations over the Brillouin zone (BZ) were performed using dense meshes of special k-points. At selected volumes, the structure was fully relaxed to the optimized configuration through the calculation of the forces on atoms and the stress tensor. In the optimized configurations, the forces on the atoms were less than $0.002 \mathrm{eV} / \AA$ and the deviations of the stress tensor from a diagonal hydrostatic form were smaller than $1 \mathrm{kbar}$ (0.1 GPa). The set of energies $E$, volumes $(\mathrm{V})$ and pressures $(P)$ obtained was fitted with an equation of state (EoS) to obtain the bulk modulus and its pressure derivative. The interatomic distances were extracted with VESTA software [75] 
Lattice-dynamic calculations of the phonon modes were carried out at the zone centre ( $\Gamma$ point) of the $\mathrm{BZ}$ with the direct force-constant approach (or supercell method) [76]. These calculations provide not only the frequency of the normal modes, but also their symmetry and their polarization vectors. This allowed us to identify the irreducible representations and the character of the phonon modes at the $\Gamma$ point.

\section{Conclusions}

In this experimental and theoretical approach to the behaviour under compression of $\beta-\mathrm{Sb}_{2} \mathrm{O}_{3}$ compound, we have confirmed the 1 st order phase transition above $15 \mathrm{GPa}$ of previous works and have proposed that the HP phase of valentinite has an orthorhombic symmetry (s.g. Pcc2) that is a low-symmetry modification of the valentinite structure. The proposed HP phase is supported by ab initio simulations, with good quality factors in the analysis of the powder XRD and with its cationic structure confirmed by single crystal XRD.

Thanks to the analysis of the pressure dependence of Ramanactive mode frequencies in valentinite and its comparison with ab initio calculations, this work provides a solution to the misunderstanding on the irreducible representation of the vibrational modes of $\beta-\mathrm{Sb}_{2} \mathrm{O}_{3}$ found in the literature. Moreover, we have presented a tentative assignment of the symmetry of the observed first-order Raman-active modes of $\beta-\mathrm{Sb}_{2} \mathrm{O}_{3}$.

A deep analysis of the compressibility of the theoretically simulated $\mathrm{SbO}_{5} E$ polyhedral unit, empty linear cavities, and the unit cell volume ( $F-f$ plots), as well as the pressure dependence of $\mathrm{Sb}$ and $\mathrm{O}$ free atomic coordinates reveals changes around 2 , 4 and $10 \mathrm{GPa}$ that correlate with changes observed in the vibrational modes of valentinite under compression. These changes were mainly observed in the HP behaviour of the Bader charge and volume around $\mathrm{Sb}$ and $\mathrm{O} 2$ and they have been interpreted as changes in the orientation and distortion of cation LEPs, respectively. In particular, the changes observed at 2 and 4 GPa can be considered as internal IPTs in valentinite, which are hidden (not evidenced in the bulk compressibility) due to the dominance of the compressibility of the voids of the structure in the bulk compressibility. The transitions at 2 and 4 GPa have been identified with an order higher than 2 because they involve a change of interaction between the neighbouring LEPs (unpaired electrons). These transitions trigger the formation of new bonds, thus increasing the Sb coordination changes from 3 at room pressure to $3+1$ above $2 \mathrm{GPa}$. Hence, they constitute a sequence of transitions from an acicular quasimolecular solid to a 3D solid.

Finally, the F-f plots exhibit a change of the compressibility of the whole rod-like structure above $10 \mathrm{GPa}$ that is consistent with the general softening of vibrational modes. We have interpreted this instability caused by the strong interaction between the central $\mathrm{O} 1$ atoms and $\mathrm{Sb}$ atoms of neighbour $\mathrm{Sb}_{2} \mathrm{O}_{3}$ units when the large $\mathrm{Sb}-\mathrm{O} 1$ distance decreases below $2.38 \AA$; this leads to an increase in coordination of $\mathrm{Sb}$ atoms to $3+1+1$ above $10 \mathrm{GPa}$. Therefore, the coordination of $\mathrm{Sb}$ clearly increases to 5 in the high-pressure phase above $15 \mathrm{GPa}$.
We hope that this work will promote further studies in valentinite for an unambiguous identification of the cause of the changes observed above $10 \mathrm{GPa}$ and the determination of its high pressure phase. We hope that this work, uncovering the interaction between unpaired LEPs in valentinite at $2 \mathrm{GPa}$, will stimulate further work in the study of subtle pressure-induced phase transitions in molecular and quasi-molecular solids, especially in compounds with cations featuring stereochemically active LEPs, like in group-XV sesquioxides, where a detailed search could lead to the discovery of new and hitherto unexpected internal transitions.

\section{Author Contributions}

Conceptualization has been performed by JAS. Wirting has been performed by JAS and FJM. Investigation on experimental methods was conducting by JAS, FJM, JR, CP, JP, VPCG, JI and VM. Investigation on theoretical calculations was done by AM, PRH, JCG and VM. Methodology was discussed by JAS and FJM.

\section{Conflicts of interest}

There are no conflicts to declare.

\section{Acknowledgements}

This research was partially supported by MINECO (FIS201783295-P, MAT2016-75586-C4-1/2/3-P, PGC2018-097520-A100, RED and PID2019-106383GB-41/42/43), by the MALTACONSOLIDER TEAM network (RED2018-102612-T) and by the EU-FEDER. JAS acknowledges Ramon y Cajal fellowship (RYC2015-17482) for financial support. VM thanks the Juan de la Cierva Program for financial support (FJCl-2016-27921). We also thank ALBA (2016091901 experiment) and DIAMOND light source (CY22223 experiment) for providing beamtime, and Mark Warren, Sarah Barnett and Dominik Daisenberger for his help in the single-crystal X-ray diffraction experiment under pressure.

\section{Notes and references}

1 J. Divya, N. J. Shivaramu, W. D. Roos, W. Purcell, H. C. Swart, Synthesis, surface and photoluminescence properties of $\mathrm{Sm}^{3+}$ doped $\alpha-B i 2 O 3$, J. Alloy and Comp., 2021, 854, 157221.

2 K. P. Lakshmi, R. Deivananyagam, M. M. Saijumon, Carbon nanotube 'wired' octahedral Sb2O3/graphene aerogel as efficient anode material for sodium and lithium ion batteries, J. Alloy and Comp. 2021, 857, 158267.

3 A. F. Gerdelidani, H. Towfighi, K. Shahbazi, D. T. Lamb, G. Choppala, S. Abbasi, A. S. M. Fazle Bari, R. Naidu, M. Mahmudur Rahman, Arsenic geochemistry and mineralogy as a function of particle-size in naturally arsenic-enriched soils, $J$. of Hazardous Mat., 2021, 403, 123931.

4 A. Matsumoto, Y. Koyama, and I. Tanaka, Structures and energetics of $\mathrm{Bi} 2 \mathrm{O} 3$ polymorphs in a defective fluorite family derived by systematic first-principles lattice dynamics calculations, Phys. Rev. B, 2010, 81, 094117. 
5 A. Matsumoto, Y. Koyama, A. Togo, M. Choi and I. Tanaka, Electronic structures of dynamically stable $\mathrm{As} 2 \mathrm{O} 3, \mathrm{Sb2O} 3$, and Bi2O3 crystal polymorphs, Phys. Rev. B, 2011, 83, 214110.

6 J. P. Allen, J. J. Carey, A. Walsh, D. O. Scanlon and G. W. Watson, Electronic Structures of Antimony Oxides, J. Phys. Chem. C, 2013, 117, 14759.

7 G. W. Watson and S. C. Parker, Origin of the Lone Pair of $\alpha$ $\mathrm{PbO}$ from Density Functional Theory Calculations, J. Phys. Chem. B, 1999, 103, 1258.

8 G. W. Watson, S. C. Parker and G. Kresse, Ab initio calculation of the origin of the distortion of $\alpha-P b O$, Phys. Rev. B, 1999, 59, 8481.

9 Q. Guo, Y. Zhao, C. Jiang, W. L. Mao, Z. Wang, J. Zhang, and Y. Wang, Pressure-Induced Cubic to Monoclinic Phase Transformation in Erbium Sesquioxide Er2O3, Inorg. Chem., 2007, 46, 6164.

10 P. A. Guńka, K. F. Dziubek, A. Gładysiak, M. Dranka, J. Piechota, M. Hanfland, A. Katrusiak and J. Zachara, Compressed Arsenolite As4O6 and Its Helium Clathrate As406.2He, Cryst. Growth Des., 2015, 15, 3740.

11 J. A. Sans, F. J. Manjón, C. Popescu, V. P. Cuenca-Gotor, O. Gomis, A. Muñoz, P. Rodríguez-Hernández, J. ContrerasGarcía, J. Pellicer-Porres, A. L. J. Pereira, D. Santamaría-Pérez, and $A$. Segura, Ordered helium trapping and bonding in compressed arsenolite: Synthesis of As406.2He, Phys. Rev. B, 2016, 93, 054102.

12 V. P. Cuenca-Gotor, O. Gomis, J. A. Sans, F. J. Manjón, A Muñoz, P. Rodríguez-Hernández, and A. Muñoz, Vibrational and elastic properties of As4O6 and As406.2He at high pressures: Study of dynamical and mechanical stability, J. Appl. Phys., 2016, 120, 155901.

13 A. L. J. Pereira, L. Gracia, D. Santamaría-Perez, R. Vilaplana, F. J. Manjon, D. Errandonea, M. Nalin, A. Beltrán, Structural and vibrational study of cubic $\mathrm{Sb} 2 \mathrm{O} 3$ under high pressure, Phys. Rev. B, 2012, 85, 174108.

14 Z. Zhao, Q. Zeng, H. Zhang, S. Wang, S. Hirai, Z. Zeng and W. L. Mao, Structural transition and amorphization in compressed a-Sb2O3, Physical Review B, 2015, 91, 184112.

15 A. L. J. Pereira, D. Errandonea, A. Beltran, L. Gracia, O. Gomis, J. A. Sans, B. García-Domene, A. Miquel-Veyrat, F. J. Manjon, A. Munoz and C. Popescu, Structural study of $\alpha-B i 2 O 3$ under pressure, J. Phys: Cond. Mat., 2013, 25, 475402.

16 E. Soignard, S. A. Amin, Q. Mei, C. J. Benmoreand J. L. Yarger, High-pressure behaviour of As203: Amorphous-amorphous and crystalline-amorphous transitions, Phys. Rev. B, 2008, 77, 144113.

17 P. A. Guńka, M. Dranka, M. Hanfland, K. F. Dziubek, A. Katrusiak and J. Zachara, Cascade of High-Pressure Transitions of Claudetite II and the First Polar Phase of Arsenic(III) Oxide, Cryst. Growth Des., 2015, 15, 3950.

18 N. Cornei, N. Tancret, F. Abraham and O. Mentre, New $\varepsilon$ Bi2O3 Metastable Polymorph, Inorg. Chem., 2006, 45, 4886.

19 A. L. J. Pereira, J. A. Sans, R. Vilaplana, O. Gomis, F. J. Manjón, P. Rodríguez-Hernández, A. Muñoz, C. Popescu and A. Beltrán, Isostructural Second-Order Phase Transition of $\beta$-Bi2O3 at High Pressures: An Experimental and Theoretical Study, J. Phys. Chem. C, 2014, 118, 23189.

20 N. C. Norman, Chemistry of arsenic, antimony and bismuth. Springer Netherlands 1998.

21 M. Brebu, E. Jakab, Y. Sakata, Effect of flame retardants and $\mathrm{Sb} 2 \mathrm{O} 3$ synergist on the thermal decomposition of high-impact polystyrene and on its debromination by ammonia treatment. J. Anal. Appl. Pyrolysis, 2007, 79, 346.

22 M. M. Si, J. Feng, J. W. Hao, L. S. Xu, J. X. Du, Synergistic flame retardant effects and mechanisms of nano-Sb2O3 in combination with aluminum phosphinate in poly (ethylene terephthalate). Polymer Degradation and Stability, 2014, 100, 70 .
23 J. H. Youk, R. P. Kambour, W. J. MacKnight, Preparation and Polymerization of Ethylene 2,6-Naphthalenedicarboxylate Cyclic Oligomers. Macromolecules, 2000, 33, 3606-3610.

24 G. Venkateshwarlu, K. C. Rajanna, P. K. Saiprakash, Antimony Trioxide as an Efficient Lewis Acid Catalyst for the Synthesis of 5-Substituted 1HTetrazoles. Synthetic Communications, 2009, 39, 426.

25 K. E. Alifantis, S. A. Hackney, K. R. Vasant, High Energy density lithium batteries: materials, engineering, applications. Wiley VCH, 2010.

26 C. M. Park, J. H. Kim, H. Kim, H. J. Sohn, Li-alloy based anode materials for Li secondary batteries. Chem. Soc. Rev., 2010, 39, 3115.

27 J. S. Zabinski, M. S. Donley, N. T. McDevitt, Mechanistic study of the synergism between $\mathrm{Sb} 2 \mathrm{O} 3$ and MoS2 lubricant systems using Raman spectroscopy. Wear, 1993, 165, 103.

28 A. Ghosh, D. Chakravorty, Transport properties of semiconducting CuO-Sb2O3-P2O5 glasses. J. Phys.: Condens. Matter, 1991, 3, 3335.

29 L. A. Gómez, C. B. de Araújo, D. N. Messias, L. Misoguti, S. C. Zilio, M. Nalin, Y. Messaddeq, Nonresonant third-order nonlinearity of antimony glasses at telecom wavelengths. J. App. Phys., 2006, 100, 116105.

30 R. Koivula, The effect of dopant's valence $(+I I I$ and $+V)$ on the anion/cation uptake properties of antimony-doped tin dioxide. Cent. Eur. J. Chem., 2010, 8, 1179.

31 K. Samanta, P. Bhattacharya, R. S. Katiyar, Raman scattering studies of p-type Sb-doped ZnO thin films. J. Appl. Phys., 2010, 108, 113501.

32 H. Y. Liu, N. Izyumskaya, V. Avrutin, Ü. Ozgür, A. B. Yankovich, A. V. Kvit, P. M. Voyles, H. Morkoc, Donor behaviour of Sb in ZnO. J. Appl. Phys., 2012, 112, 033706.

33 E. Senthil Kumar, F. Mohammadbeigi, S. Alagha, Z. W. Deng, I. P. Anderson, T. Wintschel, S. P. Watkins, Optical evidence for donor behaviour of Sb in ZnO nanowires, Appl. Phys. Lett., 2013, 102, 132105.

34 H. S. Chin, K. Y. Cheong, K. A. Razak, Review on oxides of antimony nanoparticles: synthesis, properties, and applications. J. Mater. Sci., 2010, 45, 5993.

35 A.-H. Geng, L. Cao, C. Wan, Y. Ma, High-pressure Raman investigation of the semiconductor antimony oxide, Phys. Stat. Sol. C, 2011, 8, 1708.

36 A.-H. Geng, L.-H. Cao, Y.-M. Ma, Q.-L. Cui, C.-M. Wan, Experimental Observation of Phase Transition in $\mathrm{Sb} 2 \mathrm{O} 3$ under High Pressure, Chin. Phys. Lett., 2016, 33, 097401.

37 Y. Zou, W. Zhang, X. Li, M. Ma, X. Li, C.-H. Wang, B. He, S. Wang, Z. Chen, Y. Zhao, B. Li, Pressure-induced anomalies and structural instability in compressed $\beta$-Sb2O3, Phys. Chem. Chem. Phys., 2018, 20, 11430.

38 L.D. Landau and E. M. Lifshitz, Statistical physics (Pergamon Press, London 1958), Ch. 14. p. 430.

$39 \mathrm{P}$. Ehrenfest, Phase transitions in the usual and generalized sense, classified according to the singularities of the thermodynamic potential, Proc. Roy. Acad. Amsterdam 1933, 36, 153.

40 I. M. Lifshitz, Anomalies of Electron Characteristics of a Metal in the High Pressure Region, Sov. Phys. JETP 1960, 11, 1130.

41 T. E. Jones, M. E. Eberhart, D. P. Clougherty, Topological Catastrophe and Isostructural Phase Transition in Calcium, Phys. Rev. Lett., 2010, 105, 265702.

42 R. J. Angel, J. Gonzalez-Platas, M. Alvaro, EosFit7c and a Fortran module (library) for equation of state calculations, Zeitschrift für Kristallographie, 2014, 229, 405-419.

43 It must be stressed that the BM EoS order of experimental volume data was determined by the analysis of the F-f plot of theoretical simulations (Figure S8(top) in the Supplementary Material). The observation of this plot led us to the first observation of an anomalous compressibility above $10 \mathrm{GPa}$. 
44 A. L. J. Pereira, O. Gomis, J. A. Sans, J. Contreras-García, F. J. Manjón, P. Rodríguez-Hernández, A. Muñoz, A. Beltrán, $\beta$-Bi2O3 under compression: Optical and elastic properties and electron density topology analysis, Phys. Rev. B, 2016, 93 224111.

45 CrysAlisPRO, Oxford Diffraction /Agilent Technologies UK Ltd, Yarnton, England.

46 G. M. Sheldrick, SHELXT - Integrated space-group and crystalstructure determination, Acta Cryst., 2015, A71, 3.

47 G. M. Sheldrick, Crystal structure refinement with SHELXL, Acta Cryst., 2015, C71, 3.

48 M. I. Arroyo, J. M. Perez-Mato, C. Capillas, E. Kroumova, S. Ivantchev, G. Madariaga, A. Kirov, H. Wondratschek, Bilbao Crystallographic Server I: Databases and crystallographic computing programs, Zeitschrift für Kristallographie, 2006, 221, 15.

49 C. A. Cody, L. Dicarlo, R. K. Darlington, Vibrational and thermal study of antimony oxides, Inorg. Chem., 1979, 18, 1572.

50 P. J. Miller, C. A. Cody, Infrared and Raman investigation of vitreous antimony trioxide, Spectrochim. Acta A, 1982, 38, 555.

51 A. E. Panasenko, L. A. Zemnukhova, L. N. Ignatieva, T. A. Kaidalova, S. I. Kuznetsov, N. V. Polyakova, Y. V. Marchenko, Phase composition of antimony(III) oxide samples of different origin, Inorg. Mater., 2009, 45, 402.

52 E. I. Voit, A. E. Panasenko, L. A. Zemnukhova, Vibrational spectroscopic and quantum chemical study of antimony(III) oxide, J. Struct. Chem., 2009, 50, 60.

53 R. F. W. Bader, M. A. Austen, Properties of atoms in molecules: Atoms under pressure, The Journal of Chemical Physics, 1997, 107, 4271.

54 K. Zhao, Y. Wang, Y. Sui, C. Xin, X. Wang, Y. Wang, Z. Liu, B. Li, First principles study of isostructural phase transition in Sb2Te3 under high pressure, Phys. Status Solidi RRL, 2015, 9, 379.

55 K. K. Pandey, H. K. Poswal, R. Kumar, S. M. Sharma, High pressure iso-structural phase transition in BiMn205, J. Phys. Condens. Matter, 2013, 25, 325401.

56 G. Jaegger, The Ehrenfest Classification of Phase Transitions: Introduction and Evolution, Arch. Hist. Exact Sci. 1998, 53, 51.

57 T. Ma, S. Wang, Phase Transition Dynamics. Springer New York Heidelberg Dordrecht London 2014, 10.1007/978-14614-8963-4.

58 A. Dewaele, P. Loubeyre M. Mezouar, Equations of state of six metals above 94 GPa, Phys. Rev. B, 2004, 70, 094112.

59 F. Fauth, I. Peral, C. Popescu and M. Knapp, The new Material Science Powder Diffraction beamline at ALBA Synchrotron, Powder Diffr., 2013, 28, S360.

60 A. P. Hammersley, S. O. Svensson, M. Hanfland, A. N. Fitch and D. Hausermann, Two-dimensional detector software: From real detector to idealised image or two-theta scan, High Pressure Res., 1996, 14, 235.

61 A. C. Larson and R. B. von Dreele, General Structure Analysis System (GSAS), LANL Report, 2004, 86, 748.

62 B. H. Toby, EXPGUI, a graphical user interface for GSAS, J. Appl. Crystallogr., 2001, 34, 210.

63 B. Garcia-Domene, H. M. Ortiz, O. Gomis, J. A. Sans, F. J. Manjón, A. Muñoz, P. Rodríguez-Hernández, S. N. Achary, D. Errandonea, D. Martínez-García, A. H. Romero, A. Singhaland A. K. Tyagi, High-pressure lattice dynamical study of bulk and nanocrystalline $\mathrm{In}_{2} \mathrm{O}_{3}$, J. Appl. Phys., 2012, 112, 123511.

64 Z. Sui, S. Hu, H. Chen, C. Gao, H. Su, A. Rahman, R. Dai, Z. Wang, $X$. Zheng and Z. Zhang, Laser effects on phase transition for cubic $\mathrm{Sb}_{2} \mathrm{O}_{3}$ microcrystals under high pressure, J. Mater. Chem. C, 2017, 5, 5451

65 M. K. Mao, J. Xu and P. M. Bell, Calibration of the ruby pressure gauge to 800 kbar under quasi-hydrostatic conditions, J. Geophys. Res., 1986, 91, 4673.
66 G. J. Piermarini, S. Block and J. D. Barnett, Hydrostatic limits in liquids and solids to 100 kbar, J. Appl. Phys., 1973, 44, 5377.

67 S. Klotz, J.-C. Chervin, P. Munsch and G. Le Marchand, Hydrostatic limits of 11 pressure transmitting media, J. Phys. D: Appl. Phys., 2009, 42, 075413.

68 P. Hohenberg and W. Kohn, Inhomogeneous Electron Gas, Phys. Rev., 1964, 136, B864.

69 G. Kresse and J. Hafner, Ab initio molecular dynamics for liquid metals, Phys. Rev. B, 1993, 47, 558.

$70 \mathrm{G}$. Kresse and J. Furthmüller, Efficiency of ab-initio total energy calculations for metals and semiconductors using a plane-wave basis set, Comput. Mat. Sci., 1996, 6, 15.

71 G. Kresse and J. Furthmüller, Efficient iterative schemes for $a b$ initio total-energy calculations using a plane-wave basis set, Phys. Rev. B, 1996, 54, 11169.

72 P. E. Blöchl, Projector augmented-wave method, Phys. Rev. B, 1994, 50, 17953.

73 G. Kresse and D. Joubert, From ultrasoft pseudopotentials to the projector augmented-wave method, Phys. Rev. B, 1999, 59, 1758.

74 J. P. Perdew, A. Ruzsinszky, G. I. Csonka, O. A. Vydrow, G. E. Scuseria, L. A. Constantin, X. Zhou and K. Burke, Restoring the Density-Gradient Expansion for Exchange in Solids and Surfaces, Phys. Rev. Lett., 2008, 100, 136406.

75 K. Momma and F. Izumi, VESTA 3 for three-dimensional visualization of crystal, volumetric and morphology data, J. Appl. Crystallogr., 2011, 44, 1272.

76 K. Parlinsky, Computer code PHONON (2008). URL wolf.ifj.edu.pl/phonon/ 